\title{
An E-Governance Web Security Survey
}

\author{
Deven C. Pandya ${ }^{1}$, Dr. Narendra J. Patel ${ }^{2}$ \\ ${ }^{I}$ (Department of Computer Application, U.V. Patel Engg. College/ Ganpat University, Kherva, Gujarat India) \\ 2 ((Department of Computer Application, U.V. Patel Engg. College/ Ganpat University,Kherva, Gujarat India)
}

\begin{abstract}
The purpose of the study is to explore web security status of the E-Governance websites and web applications. The central theme of the paper is to study and analyze the security vulnerabilities in the technologies utilized for E-Governance website and web application development. The study was conducted in the State of Gujarat,India. The data related to web development technologies, vulnerabilities affecting those technologies, vulnerability severity, and vulnerability type were gathered from 26 E-Governance website/Web application for detail analysis. The outcome of the study depicts the relationship between technology vis-a-vis vulnerability type and vulnerability severity.
\end{abstract}

Keywords: Web Security, Vulnerability, Risk, E-Governance

\section{Introduction}

E-Governance is the public sector's use of information and communication technologies with the aim of improving information and service delivery, encouraging citizen participation in the decision-making process and making government more accountable, transparent and effective. [1] Information security, at times, shortened to InfoSec, refers to the processes and methodologies which are designed and implemented to protect print, electronic, or any other form of confidential, private and sensitive information or data from unauthorized access, use, misuse, leak, destruction, alteration, or disruption. [2]. Information security is important for successful E-Governance implementation. Protecting confidentiality of an individual's personal data that he/she provides to obtain government services is an important challenge in implementing e-Governance. [3] Apart from E-Government implementation, effective Information Security is essential to protect Government IT assets from Cyber terrorism, Advance Persistent Threats, hackers etc.

In India, it is compulsory for each Government web sites/web application to go through security audit from enlisted agencies before hosting and after addition of new module. In addition to this department must devise a security policy to address various security issues related to website/web application [4] Government of Gujarat has also made security audit obligatory on each instance of website update or every six month whichever is earlier for all the organizations through Computer Emergency Response Team -India enlisted security auditors. [5]

\section{Web Security Survey}

Vulnerability scanners are effective tools for web security survey and finding vulnerabilities in the web application or website. Total 26 Websites/Web Applications related to Government Departments were surveyed and scanned for vulnerabilities. Accunetix scanner is utilized for this purpose. Accunetix ranked no 1 according to the 2013/2014 Web Application Vulnerability Scanners Benchmark. [6] [7] The websites/Web Application was chosen randomly from Government website directory. The websites/ web applications were scanned for parameters like Vulnerability, Vulnerability severity, Vulnerability type, Asset information, and Technology used for development. Using the survey results the research article was already published to depict the vulnerability its severity, impact and solutions in our paper on E-governance web security audit. [8] The remaining results and analysis is discussed here to gain full insights of E-Governance web security in Indian context

The Vulnerability found and their type and severity level given in Table $\mathbf{1}$ below. In each severity level group below table depicts vulnerabilities having occurrence in more than $10 \%$ websites/web applications.

\section{Technology Vs Severity analysis}

Web security scanner crawler identified mainly two technologies Microsoft and Apache during the scan. All web application/websites either hosted on Microsoft Server or Apache Server. The Apache technology here includes Apache Tomcat and Apache HTTP Server. 
An E-Governance Web Security Survey

Table 1

\begin{tabular}{|c|c|c|}
\hline Vulnerability & Severity Level & Vulnerability Type \\
\hline Cross Site Scripting & HIGH & Validation \\
\hline Proxy accepts CONNECT requests & HIGH & Validation \\
\hline ASP.NET Padding Oracle Vulnerability & HIGH & Configuration \\
\hline Microsoft IIS tilde directory enumeration & HIGH & Validation \\
\hline Application error message & MEDIUM & Informational \\
\hline fTML form without CSRF Protection-Cross Site Request & MEDIUM & Informational \\
\hline User credentials are sent in clear text & Validation \\
\hline ASP.NET error message & MEDIUM & Informational \\
\hline Session Cookie without Secure flag set & MEDIUM & Validation \\
\hline OPTIONS method is enabled & Low & Informational \\
\hline Broken Links & Low & Informational \\
\hline Password type input with autocomplete enabled & Informational & Informational \\
\hline Typical login page & Informational & Informational \\
\hline Possible internal IP address disclosure & Informational & Configuration \\
\hline
\end{tabular}

The server technology pattern depicts that software development technology like Microsoft .net framework, Java, PHP has been utilized for website/web application development. Below given Table 2 depicts relation between server Technology and Vulnerability severity.

Table 2

\begin{tabular}{|c|c|c|c|c|}
\hline Technology Vs. Severity & High & Medium & Low & Informational \\
\hline Apache & $9.84 \%$ & $27.87 \%$ & $44.26 \%$ & $18.03 \%$ \\
\hline Microsoft-IIS & $9.09 \%$ & $24.24 \%$ & $37.37 \%$ & $29.29 \%$ \\
\hline
\end{tabular}

As seen from the Table 2, in Apache technology, incidence of low severity vulnerability is highest with $44.26 \%$ followed by medium $27.87 \%$, informational $18.03 \%$ and high with $9.84 \%$ occurrence. In Microsoft Technology occurrence of low severity vulnerability is highest with $37.37 \%$ followed by medium $24.24 \%$, informational $29.29 \%$ and high with $9.09 \%$ occurrence. Here we can conclude that in both the technologies occurrence of low and medium severity level vulnerabilities is higher in comparison to high or informational severity type vulnerabilities. In addition to this, we can also notice that occurrence of medium and low vulnerabilities are higher in Apache technology than Microsoft technology while occurrences of high and informational vulnerabilities are higher in Microsoft technology than Apache technology.

Table 3

\begin{tabular}{|c|c|}
\hline Technology & Utilization \\
\hline Apache & $38.13 \%$ \\
\hline Microsoft-IIS & $61.88 \%$ \\
\hline
\end{tabular}

Table 3 depicts \% utilization of technology for website/web application development. As per the Table 3, it is clear that Microsoft Technology is utilized in $61.88 \%$ sites while Apache Technology is utilized for $38.13 \%$ websites. It means the majority of websites/web applications used Microsoft Technology for Development.

\section{Technology Vs Vulnerability Type analysis}

Below Table 4 depicts the relation between Technology and Vulnerability type.

Table 4

\begin{tabular}{|c|c|c|c|c|}
\hline Technology Vs. Vulnerability Type & Configuration & Informational & Validation & Grand Total \\
\hline Apache & $18.18 \%$ & $58.18 \%$ & $23.64 \%$ & $100.00 \%$ \\
\hline Microsoft-IIS & $12.90 \%$ & $50.54 \%$ & $36.56 \%$ & $100.00 \%$ \\
\hline
\end{tabular}

As seen from the Table 4, incidence of configuration type vulnerabilities i.e Proxy accepts CONNECT requests, Microsoft IIS tilde directory enumeration etc. and Informational type vulnerabilities i.e. Possible internal IP address disclosure, Broken Links etc. is higher in the websites using Apache Technology as compared to the websites based on Microsoft technology. The incidence of Validation type vulnerabilities i.e. Cross-Site Scripting, Application error message etc. and Informational type vulnerabilities i.e. Possible internal IP address disclosure, Broken Links etc. are higher in websites based on Microsoft Technology than websites based on Apache Technology. 


\section{Severity VS Vulnerability Type}

Below given Table 5 depicts the relation between Vulnerability severity and Vulnerability Type found in the EGovernance websites.

Table 5

\begin{tabular}{|c|c|c|c|}
\hline Severity vs. Vulnerability Type & Configuration & Informational & Validation \\
\hline High & $58.33 \%$ & $0.00 \%$ & $41.67 \%$ \\
\hline Medium & $23.08 \%$ & $30.77 \%$ & $46.15 \%$ \\
\hline Low & $3.28 \%$ & $57.38 \%$ & $39.34 \%$ \\
\hline Informational & $11.11 \%$ & $88.89 \%$ & $0.00 \%$ \\
\hline Grand Total & $14.86 \%$ & $53.38 \%$ & $31.76 \%$ \\
\hline
\end{tabular}

It is clearly evident from the results depicted in above Table 5 that major high severity vulnerabilities found in configuration type vulnerability group. Medium severity vulnerabilities mostly found in validation type vulnerability group. Low and informational severity vulnerabilities found mainly in informational vulnerability type group.

\section{Conclusion}

Among 26 Websites/Web applications Microsoft Technology is utilized in $61.88 \%$ websites while Apache Technology is utilized for $38.13 \%$ websites. From the analysis, it is observed that in both the technologies occurrence of low and medium severity level vulnerabilities is higher in comparison to high or informational severity type vulnerabilities. Apart from this, it is also observed that occurrence of medium and low vulnerabilities are higher in Apache technology than Microsoft technology while occurrences of high and informational vulnerabilities are higher in Microsoft technology than Apache technology. In technology and vulnerability type relation, it is observed that incidence of configuration type and informational type vulnerabilities is higher in the websites using Apache Technology as compared to the websites based on Microsoft technology. The incidence of Validation type vulnerabilities are higher in websites based on Microsoft Technology than websites based on Apache Technology. In severity vs. vulnerability type relation, it is observed that major high severity vulnerabilities belong to configuration type vulnerability group while medium severity vulnerabilities mostly belong to validation type vulnerability group. Low and informational severity vulnerabilities mainly exist in informational vulnerability group.

\section{References}

[1]. UNESCO, "E-Governance," UNESCO, 2001. [Online]. Available: http://portal.unesco.org/ci/en/ev.phpURL_ID=3038\&URL_DO=DO_TOPIC\&URL_SECTION=201.html. [Accessed 14 July 2016].

[2]. Sans. Institute, "Information Security Resource," SANS, [Online]. Available: https://www.sans.org/information-security/. [Accessed 14 July 2016].

[3]. P. Mittal and A. Kaur, "E-Governance - A challenge for India," International Journal of Advanced Research in Computer Engineering \& Technology (IJARCET), vol. 2, no. 3, March 2013.

[4]. "Guidelines for Indian Government Websites," Department of Administrative Reforms and Public Grievances, Government of India, 2009.

[5]. "Guidelines for Registration, Hosting and periodic security audit of Government Websites," Science and Technology Department of Government of Gujarat, Gandhinagar, 2014.

[6]. S. Chen, "Security Tools Benchmarking," [Online]. Available: ] http://sectooladdict.blogspot.in/2014/02/wavsep-web-applicationscanner.html.

[7]. "Web Application Scanner Comparision," Acunetix, [Online]. Available: https://www.acunetix.com/blog/news/acunetixcomparison-web-application-scanners/.

[8]. "Security Innovation Appsec. Blog," Security Innovation, Inc. , [Online]. Available: http://web.securityinnovation.com/appsecweekly/blog/bid/89728/Prevent-Information-Disclosure-in-Error-Messages. 